\title{
On-Line Accuracy Assessment for the Dual Six-Port ANA: Extension to Nonmating Connectors
}

\author{
CLETUS A. HOER, MEMBER, IEEe, AND GLENN F. ENGEN, FELLOW, IEEE
}

\begin{abstract}
A technique is described for calibrating a dual six-port or four-port automatic network analyzer (ANA) so that the scattering parameters of two-port devices having any combination of connectors can be measured. The technique is a generalization of the "thru-reflectline" (TRL) calibration technique in which the thru is replaced with a second length of precision transmission line. Expressions for errors associated with the second line are derived.
\end{abstract}

\section{INTRODUCTION}

$\mathrm{T}$ HE "thru-reflect-line" (TRL) technique [1] for calibrating a dual six-port or four-port reflectometer has been modified so that the scattering parameters of twoport devices having any combination of connectors can be measured.

The thru, reflect, and line measurements made in the TRL calibration are shown in Fig. 1(a). The complex sidearm ratios $w_{1}$ and $w_{2}$ are measured for the three measurement conditions shown. The test ports are connected together, then one or more highly reflecting terminations are connected to one test port and then to the other test port. Finally, a length of precision transmission line is connected between the two test ports. For a dual six-port, $w_{1}$ and $w_{2}$ are each obtained from four power measurements made on the four sidearms of each six-port [1]. For a dual four-port, $w_{1}$ and $w_{2}$ are each obtained from a detector which measures the complex sidearm ratio directly.

The TRL calibration yields the parameters $A_{1}, B_{1}$, and $C_{1}$ of four-port $A$, and $A_{2}, B_{2}$, and $C_{2}$ of four-port $B$ which relate $w_{1}$ and $w_{2}$ to $\Gamma_{1}$ and $\Gamma_{2}$, respectively, as shown in Fig. 1(a). Also obtained from the TRL solution are the reflection coefficients of all the terminations used in the reflect measurements, and $\gamma l$ of the precision transmission line used in the line measurement, where $\gamma$ is the propagation constant of the line, and $l$ is its physical length. As shown in Fig. 1(a), the TRL technique can be applied only to reflectometers having identical sexless connectors at the test ports. If the requirement for making a thru connection is replaced by a measurement with a short length of line as shown in Fig. 1(b), then the calibration technique can be applied to a pair of reflectometers having identical connectors of any type, not just sexless connectors. This line-reflect-line (LRL) calibration technique is the subject of this paper.

Manuscript received June 23, 1987.

The authors are with the National Bureau of Standards, Boulder, CO 80303.

IEEE Log Number 8613604.

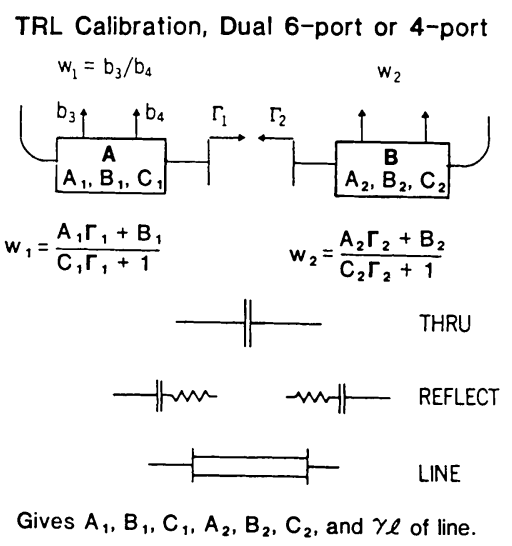

(a)

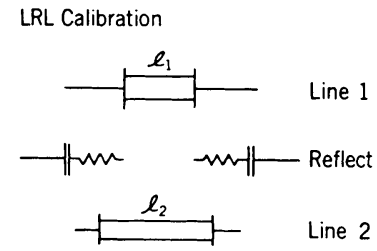

(b)

Fig. 1. (a) TRL technique for calibrating a dual six-port or four-port reflectometer. (b) LRL calibration technique where the thru is replaced with a short length of line.

\section{LRL EXAMPLES}

\section{A. Two Pairs of Lines}

An example of the LRL calibration technique is shown in Fig. 2(a) where both test port connectors are identical male connectors. When the calibration using two lines and one or more terminations with female connectors is complete, the $S$-parameters of two-port devices that have female connectors on both ends can be measured.

Fig. 2(b) shows an example where adapters have been added to obtain two female test port connectors. The LRL calibration can be applied to these new test ports using lines and terminations with male connectors. Then twoport devices that have male connectors on both ends can be measured.

To measure two-port devices that have one male and one female connector, adapter $C$ ( or $D$ ) is removed to obtain the test port configuration shown in Fig. 2(c). The parameters for reflectometer $A$ are known from the calibration in Fig. 2(a), and the parameters for reflectometer configuration $F$ are known from the calibration in Fig. 2(b). The repeatability of the connection between $A$ and 
LRL is valid for any connector.

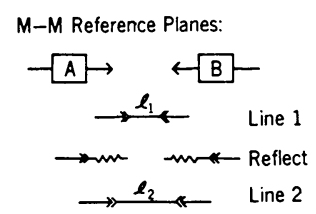

(a)

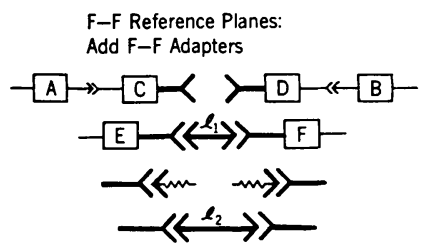

(b)

M-F Reference Planes:

Remove Adapter C (or D)

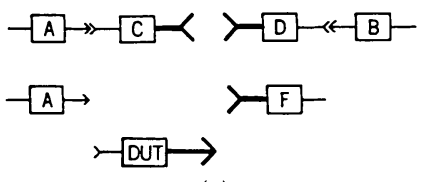

(c)

Fig. 2. (a)-(c) Using a set of female standards and a set of male standards to do a complete LRL calibration so that a two-port device with any combination of connectors can be measured.

adapter $C$ (or between $B$ and $D$ ) is not important when this sequence of measurements is used.

The connector types used in Fig. 2(a) and (b) can be completely different. For example, those in Fig. 2(a) could be type $N$, while the connectors in Fig. 2(b) could be SMA. Two-ports that have a female type $N$ on one end and a male SMA on the other end can be measured as in Fig. 2(c). The LRL calibration technique can also be applied if one set of connectors is waveguide, and the other set is coax.

\section{B. One Pair of Lines}

Only one pair of lines is needed to calibrate the two reflectometers if the connectors on the two-port under test are of the same type. For example, to measure two-port devices that have any combination of type- $N$ connectors, the reflectometers are first calibrated with type- $N$ male connectors as shown in Fig. 3(a). Then a female-female type- $N$ adapter $C$ is measured as shown in Fig. 3(b), and left connected to one of the reflectometers, say to $A$. Twoport devices that have male-female type- $N$ connectors can now be measured between $C$ and $B$. To measure two-port devices with two male type- $N$ connectors, one may remove adapter $C$ and measure the parameters of another similar adapter $D$. As shown in Fig. 3(c), leaving adapter $C$ on reflectometer $A$, and reconnecting adapter $D$ to reflectometer $B$ provides a pair of female test ports between which two-port devices that have male connectors can be measured.

\section{LRL Calibration}

The computations and software used in the TRL solution can also be used in the LRL solution with only slight

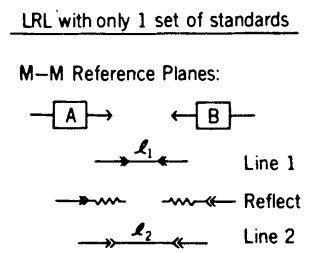

(a)

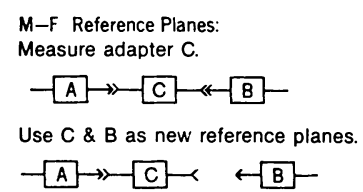

(b)

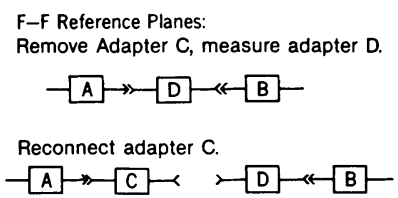

(c)

Fig. 3. (a)-(c) Using one set of standards to do a complete LRL calibration when the connectors on the device under test are the same type (but any combination of sexes).

modifications. If a line of length $l_{1}$ is used in the TRL calibration instead of a thru $\left(l_{1}=0\right)$ connection, the following modifications to the four-port parameters are obtained (see Appendix B):

$$
\begin{aligned}
B_{1} & =B_{1 t} \\
C_{1} / A_{1} & =C_{1 t} / A_{1 t} \\
B_{2} & =B_{2 t} \\
C_{2} / A_{2} & =C_{2 t} / A_{2 t} \\
A_{1} & =A_{1 t} e^{\gamma / l_{1}} \\
A_{2} & =A_{2 t} e^{\gamma / l_{1}} \\
\gamma_{2} l_{2}-\gamma_{1} l_{1} & =(\gamma l)_{t} .
\end{aligned}
$$

The terms on the left of each equation are the desired parameters. The corresponding parameters on the right of each equation with a subscript $t$ are those obtained from the TRL solution. The first four equations show that the four-port parameters $B_{1}, C_{1} / A_{1}, B_{2}$ and $C_{2} / A_{2}$ are exactly equal to those obtained from the TRL solution. Equations (5) and (6) show that the four-port parameters $A_{1}$ and $A_{2}$ are scaled by $e^{\gamma / l_{1}}$.

In the TRL calibration, the optimum electrical length of the line is $90^{\circ}$ ( or an odd multiple of $90^{\circ}$ ). Electrical lengths near $0^{\circ}$ or $180^{\circ}$ (or even multiples of $90^{\circ}$ ) must be avoided or the solution becomes ill conditioned. Equation (7) shows that when two lines are used, it is the difference in the electrical lengths of the two lines that is optimally $90^{\circ}$ and that must not be near $0^{\circ}$ or $180^{\circ}$.

\section{LRL For SeXless Connectors}

At higher frequencies the optimum length of line for the TRL calibration can become physically too short to be 
practical. However, if two lines are used, $l_{1}$ can be some convenient length and $l_{2}$ slightly longer so that the difference in length is electrically $90^{\circ}$ in the center of the frequency band. For this reason, one may want to use the LRL calibration even for sexless connectors at higher frequencies.

\section{Equivalent Reference Plane}

Equations (1)-(6) lead to

$$
\begin{aligned}
& \Gamma_{1}=e^{-\gamma_{1} l_{1}} \Gamma_{t_{1}} \\
& \Gamma_{2}=e^{-\gamma_{1} l_{1}} \Gamma_{t_{2}}
\end{aligned}
$$

which say that the reflection coefficient of each termination used in the reflect measurements, or any other $\Gamma$ measurement, will differ from $\Gamma_{t_{1}}$ or $\Gamma_{t_{2}}$ by the factor $e^{-\gamma_{1} l_{1}}$ where $\Gamma_{t_{1}}$ and $\Gamma_{t_{2}}$ are the reflection coefficients obtained from $A_{1 t}, B_{1 t}$, and $C_{1 t}$ at test port 1 , or $A_{2 t}, B_{2 t}$, and $C_{2 t}$ at test port 2 .

Equation (8) shows that $\Gamma$ ratios can be measured at either test port without knowing $\gamma_{1} l_{1}$. If (8) is compared to the following equation for transforming $\Gamma_{L}$ through a nonreflecting line of length $l$

$$
\Gamma=e^{-2 \gamma l} \Gamma_{L}
$$

we see that the effective reference plane of $\Gamma_{t}$ is at $l_{1} / 2$ which is at the center of the line of length $l_{1}$. This location agrees with our intuition as to where the reference plane should be since TRL assumes $l_{1}=0$.

\section{Determining $\gamma_{1} l_{1}$}

To complete the LRL calibration, $\gamma_{1} l_{1}$ must be determined. Four methods for determining $\gamma_{1} l_{1}$ are outlined below.

\section{A. From $l_{1}$ and $l_{2}$}

If the lines $l_{2}$ and $l_{1}$ are made from the same stock and their cross-sectional dimensions are sufficiently the same, we can assume that

$$
\gamma_{2}=\gamma_{1}
$$

Then (7) becomes

$$
\gamma_{1}\left(l_{2}-l_{1}\right)=(\gamma l)_{t}
$$

which gives

$$
\gamma_{1} l_{1}=K(\gamma l)
$$

where

$$
K \equiv \frac{l_{1}}{l_{2}-l_{1}}
$$

The physical lengths $l_{1}$ and $l_{2}$ of the two lines can be measured to obtain a value for $K$.

The uncertainty in $\gamma_{1} l_{1}$ due to the assumption that $\gamma_{2}=$ $\gamma_{1}$ can be determined as follows. Let

$$
\gamma_{2}=\gamma_{1}+\Delta \gamma
$$

Substituting (14) in (7) and solving for $\gamma_{1} l_{1}$ gives

$$
\gamma_{1} l_{1}=K(\gamma l)_{t}-K \Delta \gamma l_{2}
$$

or

$$
\gamma_{1} l_{1}=K^{\prime}(\gamma l)_{t}
$$

where

$$
K^{\prime}=K\left(1-\frac{\Delta \gamma l_{2}}{(\gamma l)_{t}}\right)
$$

Comparing (15)-(17) with (12)-(13) shows that the last term in (15) or (17) represents the error in $\gamma_{1} l_{1}$ when $\gamma_{2}$ $\neq \gamma_{1}$.

\section{B. From a Known $\Gamma$}

Another way of obtaining $\gamma_{1} l_{1}$ is from (8). If $\Gamma$ is the known reflection coefficient of a highly reflecting termination connected to either test port 1 or 2 , then (8) gives

$$
\gamma_{1} l_{1}=\log _{e} \frac{\Gamma_{t}}{\Gamma} .
$$

A matched termination cannot be used because if $\Gamma$ and $\Gamma_{t}$ are both zero, $\gamma_{1} l_{1}$ cannot be determined.

\section{From $\psi$ of $\Gamma$}

If the highly reflecting termination is a short or an offset short with a precisely known phase angle $\psi_{s}$, then $\gamma_{1} l_{1}$ can be determined from $\psi_{s}$ alone. $\left|\Gamma_{s}\right|$ need not be known. To show this, define the real and imaginary parts of $(\gamma l)_{t}$ as

$$
(\gamma l)_{t}=x+j y .
$$

Then substituting (16) and (19) in (8) gives

$$
\left|\Gamma_{s}\right| e^{j \psi_{s}}=e^{-K^{\prime} x} e^{-j K^{\prime} y}\left|\Gamma_{t}\right| e^{j \psi_{t}} .
$$

From (17) we see that $K^{\prime}$ is real if $\Delta \gamma=0$ or if $\Delta \gamma /(\gamma l)_{t}$ is real. From (14) and (7)

$$
\frac{\Delta \gamma}{(\gamma l)_{t}}=\frac{\gamma_{2}-\gamma_{1}}{\gamma_{2} l_{2}-\gamma_{1} l_{1}}=\frac{1-\gamma_{1} / \gamma_{2}}{l_{2}-l_{1} \gamma_{1} / \gamma_{2}} .
$$

Although $\gamma_{1}$ and $\gamma_{2}$ are complex, the ratio $\gamma_{1} / \gamma_{2}$ will be real (or very nearly so) if the two lines are made from the same stock. Then $K^{\prime}$ is real and (20) expands into the following two equations:

$$
\begin{aligned}
\left|\Gamma_{s}\right| & =e^{-K^{\prime} x}\left|\Gamma_{t}\right| \\
\psi_{s} & =-K^{\prime} y+\psi_{t} .
\end{aligned}
$$

Since $(\gamma l)_{t}$ is mostly imaginary, $y$ is known much more accurately than $x$. For this reason we choose (23) to calculate $K^{\prime}$

$$
K^{\prime}=\frac{\psi_{t}-\psi_{s}}{y}
$$

Then $\left|\Gamma_{s}\right|$ can be calculated from (22). Thus only the phase angle $\psi_{s}$ of the short needs to be known. For a flat short

$$
\psi_{s}=180-12 f \delta \mathrm{deg}
$$


where $f$ is the frequency in gigahertz and $\delta$ is the skin depth in centimeters into the face of the short [2]. Equations for the phase angle of an offset short [3] or an opencircuit termination [4] are given in the literature.

\section{From $\Gamma$ Ratio}

A fourth way of obtaining $\gamma_{1} l_{1}$ is from the ratio of two $\Gamma$ measurements. Measure a highly reflecting termination with unknown reflection coefficient $\Gamma_{L}$ on one of the test ports of the automatic network analyzer (ANA). Then from (8) the reflection coefficient at that test port can be written

$$
\Gamma=\Gamma_{L}=e^{-\gamma_{1} l_{1}} \Gamma_{t} .
$$

If line $l_{1}$ is then inserted between the test port and $\Gamma_{L}$, (8) gives for this second measurement

$$
\Gamma^{\prime}=e^{-2 \gamma_{1} l_{1}} \Gamma_{L}=e^{-\gamma_{1} l_{1}} \Gamma_{t}^{\prime} .
$$

Taking the ratio of (27) to (26) gives

$$
\frac{\Gamma^{\prime}}{\Gamma}=e^{-2 \gamma_{1} l_{1}}=\frac{\Gamma_{t}^{\prime}}{\Gamma_{t}}
$$

Since $\Gamma_{t}^{\prime} / \Gamma_{t}$ is known, $\gamma_{1} l_{1}$ can be calculated from this equation.

\section{UNCERTAINTY IN $\Gamma$}

Elsewhere in this TRANSACTIONS [5], [6] techniques are described for propagating random and systematic errors through the six-port calibration and measurement process when the TRL calibration technique is used. When the LRL calibration technique is used, these errors apply directly to $\Gamma_{t}$ but not to $\Gamma$. Differentiating (8) leads to the following expression for the worst-case uncertainty $d \Gamma_{i}$ in $\Gamma_{i}$ at test port $i$.

$$
d \Gamma_{i}=e^{-\gamma_{1} l_{1}} d \Gamma_{t i}-\Gamma_{i} d\left(\gamma_{1} l_{1}\right), \quad i=1,2 .
$$

where $d \Gamma_{t i}$ is the uncertainty in $\Gamma_{t i}$ obtained from propagating errors through the TRL calibration and the measurement process, and $d\left(\gamma_{1} l_{1}\right)$ is the uncertainty in $\gamma_{1} l_{1}$. As $\Gamma$ approaches zero, the uncertainty in $\Gamma$ due to $d\left(\gamma_{1} l_{1}\right)$ also approaches zero. This means that the error in $\gamma_{1} l_{1}$ causes negligible additional error in the measurement of low reflecting terminations.

Equations for estimating $d\left(\gamma_{1} l_{1}\right)$ are given in Appendix A. These equations lead to the conclusion that method $d$ in Section VI is probably the most accurate method for determining $\gamma_{1} l_{1}$.

Once the errors associated with $\Gamma_{1}$ and $\Gamma_{2}$ at both test ports are determined, the errors in the $S$-parameters of any two-port measured between these test ports are readily calculated [5].

A special case occurs when the two-port being measured is an adapter such as the female-female adapter $C$ in Fig. 3(b). In this case we are not so much interested in the parameters and errors associated with the adapter $C$, but in the parameters and errors associated with the combination of error box $A$ and adapter $C$. One way to determine directly the parameters $A_{1}^{\prime}, B_{1}^{\prime}$, and $C_{1}^{\prime}$ of the combination $A+C$, and the errors associated with these parameters is to consider $A+C$ as a new two-port be-

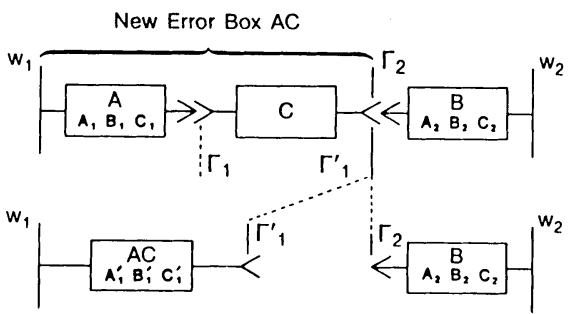

Fig. 4. Considering adapter $C$ and error box $A$ as a new two-port error box between measurement planes $w_{1}$ and $\Gamma_{2}$ to get the parameters $A_{1}^{\prime}, B_{1}^{\prime}$, and $C_{1}^{\prime}$ of $A C$ directly.

tween measurement planes $w_{1}$ and $\Gamma_{2}$ as shown in Fig. 4 . The parameters $A_{1}^{\prime}, B_{1}^{\prime}$, and $C_{1}^{\prime}$ are readily obtained from $w_{1}$ and $\Gamma_{2}$. Since the uncertainties associated with $w_{1}$ and $\Gamma_{2}$ have been determined in the TRL calibration, the uncertainties of the parameter $A_{1}^{\prime}, B_{1}^{\prime}$, and $C_{1}^{\prime}$ are also readily determined in the same way as for a two-port inserted between measurement planes 1 and 2 .

\section{Optimum Line Lengths}

The first line of length $l_{1}$ can be any convenient length; however, it should not be longer than necessary. As explained in Section III, the length $l_{2}$ of the second line should be such that the difference in phase shift $\Delta \phi$ through the two lines is $90^{\circ}$ at the center of the operating frequency range $f_{1}$ to $f_{2}$. This condition when applied to coaxial lines leads to [7]

$$
l_{2}-l_{1}=\frac{15}{f_{1}+f_{2}} \mathrm{~cm}
$$

where $f_{1}$ and $f_{2}$ are in gigahertz. The corresponding differences in phase shifts $\Delta \phi_{f_{1}}$ and $\Delta \phi_{f_{2}}$ at $f_{1}$ and $f_{2}$ are given by

$$
\Delta \phi_{f_{1}}=180-\Delta \phi_{f_{2}}=\frac{180}{1+f_{2} / f_{1}} \mathrm{deg} .
$$

If the ratio $f_{2} / f_{1}$ is so large that $\Delta \phi_{f_{1}}$ becomes too small, say less than $20^{\circ}$, then the range $f_{1}$ and $f_{2}$ may be broken into two ranges $f_{1}$ to $f_{i}$ and $f_{i}$ to $f_{2}$, where $f_{i}$ is an intermediate frequency chosen such that

$$
\frac{f_{2}}{f_{i}}=\frac{f_{i}}{f_{1}}=\sqrt{\frac{f_{2}}{f_{1}}} .
$$

Three lines are required to cover these two ranges. The lengths $l_{1}, l_{2}$, and $l_{3}$ of the three lines must satisfy

$$
l_{2}-l_{1}=\frac{15}{f_{i}+f_{2}}
$$

and

$$
l_{3}-l_{1}=\frac{15}{f_{1}+f_{i}}=\sqrt{\frac{f_{2}}{f_{1}}}\left(l_{2}-l_{1}\right) .
$$

Lengths $l_{1}$ and $l_{3}$ are used from $f_{1}$ to $f_{i}$, and lengths $l_{1}$ and $l_{2}$ are used from $f_{i}$ to $f_{2}$. The corresponding differences in phase shift are obtained from (31) using the appropriate frequencies.

Equations similar to (30) and (31) can be derived for a 
TABLE 1

Optimum Lengths For Rectangui.ar Wavfguide

\begin{tabular}{crrrrr}
\hline \hline $\begin{array}{l}\text { WAVEGUIDE } \\
\text { SIZE, WR }\end{array}$ & $\begin{array}{c}\text { CUTOFF } \\
\text { FREQ }\end{array}$ & $\begin{array}{c}\mathbf{f}_{1} \\
\text { GHz }\end{array}$ & $\begin{array}{c}\mathbf{f}_{2} \\
\mathrm{GHz}\end{array}$ & $\begin{array}{c}\ell_{2} \cdot l_{1} \\
\mathrm{Cm}\end{array}$ & $\begin{array}{c}\Delta \phi_{\mathrm{f} 1} \\
\text { DEG }\end{array}$ \\
284 & 2.078 & 2.600 & 3.950 & 3.048 & 57.1 \\
187 & 3.152 & 3.950 & 5.850 & 2.052 & 58.6 \\
137 & 4.301 & 5.850 & 8.200 & 1.370 & 65.2 \\
112 & 5.259 & 7.050 & 10.000 & 1.136 & 64.0 \\
90 & 6.556 & 8.200 & 12.400 & 0.971 & 57.4 \\
62 & 9.486 & 12.400 & 18.000 & 0.644 & 61.7 \\
42 & 14.047 & 18.000 & 26.500 & 0.445 & 60.1 \\
28 & 21.081 & 26.500 & 40.000 & 0.300 & 57.7 \\
22 & 26.341 & 33.000 & 50.000 & 0.240 & 57.4 \\
19 & 31.357 & 40.000 & 60.000 & 0.197 & 58.8 \\
15 & 39.863 & 50.000 & 75.000 & 0.160 & 58.0 \\
10 & 59.010 & 75.000 & 110.000 & 0.108 & 59.9 \\
7 & 90.840 & 110.000 & 170.000 & 0.073 & 54.3 \\
4 & 137.520 & 170.000 & 260.000 & 0.047 & 56.1 \\
3 & 173.280 & 220.000 & 325.000 & 0.037 & 59.4 \\
& & & & & \\
\hline
\end{tabular}

Optimum difference $l_{2}-l_{1}$ in two lengths of waveguide are calculated from (38) to have a phase difference $\Delta \phi$ ( or $180-\Delta \phi)$ equal to or greater than $\Delta \phi_{f_{1}}$ over the complete frequency band from $f_{1}$ to $f_{2}$. The shorter length $l_{1}$ can be any convenient length including zero.

rectangular waveguide as follows. The phase shift through a rectangular waveguide can be written [8]

$$
\phi=12 l \sqrt{f^{2}-f_{c}^{2}} \mathrm{deg}
$$

where $f$ is the frequency in gigahertz, $l$ is the physical length in centimeters, and $f_{c}$ is the cutoff frequency in gigahertz. For two lines, the difference in phase is

$$
\Delta \phi=12\left(l_{2}-l_{1}\right) \sqrt{f^{2}-f_{c}^{2}} \mathrm{deg} .
$$

Choose the lengths of line so that the differences in phase shift $\Delta \phi_{f_{1}}$ and $\Delta \phi_{f_{2}}$ at the band edges $f_{1}$ and $f_{2}$ satisfy

$$
\Delta \phi_{f_{1}}=180-\Delta \phi_{f_{2}} .
$$

This condition keeps the phase differences equally as far from $0^{\circ}$ at $f_{1}$ as it is from $180^{\circ}$ at $f_{2}$. Substituting (36) in (37) and solving for $l_{2}-l_{1}$ gives

$$
l_{2}-l_{1}=\frac{15}{\sqrt{f_{1}^{2}-f_{c}^{2}}+\sqrt{f_{2}^{2}-f_{c}^{2}}} \mathrm{~cm} .
$$

The difference in phase shifts at $f_{1}$ and $f_{2}$ are obtained by substituting (38) in (36)

$$
\Delta \phi_{f_{1}}=180-\Delta \phi_{f_{2}}=\frac{180}{1+\sqrt{\frac{f_{2}^{2}-f_{c}^{2}}{f_{1}^{2}-f_{c}^{2}}}} \mathrm{deg} .
$$

Table I gives values of $l_{2}-l_{1}$ and $\Delta \phi_{f_{1}}$ for different waveguide sizes.

\section{Conclusion}

Considerable effort has been expended by the National Bureau of Standards (NBS) to develop the TRL calibration techniques to provide on-line estimates of the random and systematic errors associated with all of the six-port calibration parameters and the measured values of $\Gamma$ or $S$ parameters. Currently, this technique has applied only to sexless connectors. By replacing the TRL thru connection with a short length of line whose $\gamma_{1} l_{1}$ is determined by one of the four methods outlined in this paper, it is possible to use all of the TRL software to calibrate a dual sixport or four-port with any type of connector. Thus only one set of software is needed for any type of connector.

\section{APPENDIX A}

The uncertainty $d\left(\gamma_{1} l_{1}\right)$ in $\gamma_{1} l_{1}$ depends on how $\gamma_{1} l_{1}$ is determined. The worst-case uncertainties associated with each of the four methods considered in Section VI for determining $\gamma_{1} l_{1}$ are derived in this appendix.

\section{A. From $l_{1}$ and $l_{2}$}

Differentiating (15) and keeping only first-order terms leads to the following expression for the uncertainty in $\gamma_{1} l_{1}$ when $\gamma_{1} l_{1}$ is determined from the physical lengths $l_{1}$ and $l_{2}$.

$$
d\left(\gamma_{1} l_{1}\right)=(\gamma l)_{t} d K+K d(\gamma l)_{t}-K l_{2} \Delta \gamma .
$$

The uncertainty $d(\gamma l)_{t}$ in $(\gamma l)_{t}$ is determined from the TRL solution [5], [6]. From (13)

$$
d K=K^{2} \frac{l_{2}}{l_{1}}\left(\frac{d l_{1}}{l_{1}}-\frac{d l_{2}}{l_{2}}\right)
$$

where $d l_{1}$ and $d l_{2}$ are the uncertainties in measuring $l_{1}$ and $l_{2}$.

\section{B. From a Known $\Gamma$}

When a known reflection coefficient is used to obtain $\gamma_{1} l_{1},(18)$ gives

$$
d\left(\gamma_{1} l_{1}\right)=\frac{d \Gamma_{t}}{\Gamma_{t}}-\frac{d \Gamma}{\Gamma} .
$$

In this expression $d \Gamma$ is the total uncertainty with which $\Gamma$ is known. 


\section{From $\psi$ of $\Gamma$}

When $\gamma_{1} l_{1}$ is determined from the known phase angle of a termination using (16) and (24), $d\left(\gamma_{1} l_{1}\right)$ is given by differentiating (16) to get

$$
d\left(\gamma_{1} l_{1}\right)=(\gamma l)_{t} d K^{\prime}+K^{\prime} d(\gamma l)_{t}
$$

where from (24)

$$
d K^{\prime}=\frac{1}{y}\left(d \psi_{t}-d \psi_{s}-K^{\prime} d y\right)
$$

Combining (A4) and (A5)

$$
\begin{aligned}
\dot{d}\left(\gamma_{1} l_{1}\right)= & \frac{(\gamma l)_{t}}{y}\left(d \psi_{t}-d \psi_{s}\right) \\
& +K^{\prime}\left(d(\gamma l)_{t}-(\gamma l)_{t} \frac{d y}{y}\right) .
\end{aligned}
$$

Let

$$
d(\gamma l)_{t}=d x+j d y .
$$

Then, since $x<y$, (A6) reduces approximately to

$$
d\left(\gamma_{1} l_{1}\right) \simeq K^{\prime} d x+j\left(d \psi_{t}-d \psi_{s}\right) .
$$

\section{From $\Gamma$ Ratio}

If $\gamma_{1} l_{1}$ is obtained by measuring a termination with and without the line $l_{1}$ inserted in front of it, the uncertainty in $\gamma_{1} l_{1}$ obtained from (28) is

$$
\begin{aligned}
d\left(\gamma_{1} l_{1}\right) & =\frac{1}{2}\left(\frac{d \Gamma_{t}}{\Gamma_{t}}-\frac{d \Gamma_{t}^{\prime}}{\Gamma_{t}^{\prime}}\right) \\
& \simeq \frac{d \Gamma_{t}}{\Gamma_{t}}=\frac{d\left|\Gamma_{t}\right|}{\left|\Gamma_{t}\right|}+j d \psi_{t} .
\end{aligned}
$$

\section{E. Comparing Methods}

Assuming that all errors are roughly the same magnitude, (A1), (A3), (A8), and (A10) imply that method D is more accurate than method B or C. Method A is probably the least accurate, but the easiest to apply.

\section{APPENDix B}

The derivation of (1)-(7) is the same as that given by Engen and Hoer [1] except that the identity matrix which describes the cascading parameters of the thru connection in (20) of that paper is replaced with

$$
R_{l_{1}}=\left[\begin{array}{cc}
e^{-\gamma_{1} / 1} & 0 \\
0 & e^{\gamma_{1} l_{1}}
\end{array}\right]
$$

and the cascading matrix for the single line is replaced with

$$
R_{/ 2}=\left[\begin{array}{cc}
e^{-\gamma_{2} / 2} & 0 \\
0 & e^{\gamma_{2} / 2}
\end{array}\right]
$$

in (21) and (25) of that paper. The rest of the derivation is the same and leads to (1)-(7) of this paper.

\section{REFERENCES}

[1] G. F. Engen and C. A. Hoer, “' 'Thru-reflect-line': An improved technique for calibrating the dual six-port ANA," IEEE Trans. Microwave Theory Tech., vol. MTT-27, pp. 987-993, Dec. 1979.

[2] P. I. Somlo, "Recession depth in metalic conductors at low frequencies," Electron. Lett., pp. 776-777, Dec. 1971.

[3] H. B. Sequeira and B. C. Yates, "Approach for evaluating effects of wall losses on quater-wave short-circuit impedance standards," IEEE Trans. Microwave Theory Tech., vol. MTT-33, pp. 1106-1109, Nov. 1985.

[4] B. Bianco et al., "Open-circuited coaxial lines as standards for microwave measurements," Electron. Lett., pp. 373-374, May 1980.

[5] R. M. Judish, "On-line accuracy assessment for the dual six-port ANA: Statistical methods for random errors," pp. 507-513, this issue.

[6] C. A. Hoer, "On-line accuracy assessment for the dual six-port ANA: Treatment of systematic errors," pp. 514-519, this issue.

[7] C. A. Hoer, "Choosing line lengths for calibrating network analyzers," IEEE Trans. Microwave Theory Tech., vol. MTT-31, pp. 7678, Jan. 1983.

[8] S. Ramo, J. Whinnery, and T. Van Dużer, Fields and Waves in Communication Electronics. New York: Wiley, 1956, p. 402. 